\title{
MODULAR ROBOTICS AS A TOOL FOR EDUCATION AND ENTERTAINMENT
}

\author{
Jacob Nielsen Henrik Hautop Lund \\ Maersk Mc-Kinney Moller Institute, University of Southern Denmark \\ Campusvej 55, 5230 Odense M \\ raider@mip.sdu.dk,hhl@mip.sdu.dk
}

\begin{abstract}
We developed I-BLOCKS, a modular electronic building block system and here we show how this system has proven useful, especially as an educational tool that allows hands-on learning in an easy manner. Through user studies we find limitations of the first I-BLOCKS system, and we show how the system can be improved by introducing a graphical user interface for authoring the contents of the individual I-BLOCK, by developing a new cubic block shape with new physical and electrical connectors, and by including new embedded electronics. We developed and evaluated the IBLOCKS as a manipulative technology through studies in both schools and hospitals, and in diverse cultures such as in Denmark, Finland, Italy and Tanzania.
\end{abstract}

\section{KEYWORDS}

Educational technology, constructionism, educational robots, entertainment robots, developing countries.

\section{INTRODUCTION}

Miniaturisation of computing makes it possible to employ new educational practises and revolutionise our idea about programming through the use of technological components. With the development of physical, intelligent building blocks (e.g. neural building blocks (Lund, 2003b)), it is investigated how it may become possible to 'program by building'. The construction with intelligent building blocks results not only in the development of a physical structure, but also in the development of a functionality of the physical structure. So construction of functionality can happen with physical building blocks that each contains computational processing and communication. After the basic development of the first hardware realisations, we worked on applying our I-BLOCKS technology in the fields of psychology, education and interaction design. We believe that the I-BLOCKS are important tools for constructionism, since they allow easy, hands-on manipulation with both physical and functional structures, e.g. for "playing" with cognitive tasks.

Other researchers (e.g. Ishii and Ulmer, 1997) have presented haptic and tangible interfaces where physical objects are coupled with digital representations. Any change in the physical arrangement is recognized and interpreted as a controlling action for the digital counterpart, e.g. as in the navigational blocks (Camarata et al., 2002) that provide visitors of a virtual museum with a direct manipulation experience using physical blocks to navigate a data space. Also, the Tangible Computation Bricks (McNerney, 2001) are physical building blocks augmented with embedded micro-processors that implement a programming language for scientific exploration. However, different from the I-BLOCKS presented here, in McNerney's implementation, building blocks can only stack in one dimension, and they allow only for sequential control. Whereas this may be suitable in some cases, in other cases a more general approach allowing two or three dimensional construction and parallel control may be suitable, see I-BLOCKS experiments below. Indeed, in this paper we will put focus of the technological development towards 3D building possibilities.

Most of the currently available prototypes of tangible interfaces share the characteristic to specify a computation that is performed by a target system. In a sense the tangible interface is still separated by any produced output either in the physical or in the virtual world. However, with the I-BLOCKS technology, we 
try to take a step ahead w.r.t. other existing implementations of building blocks, developing building devices able to simultaneously perform computations and to act as output devices of the intended functionality. They are not control systems but both input and output devices that are constructed by the users. Therefore, the IBLOCKS objective is to develop a concept of seamless interface to manipulate physical objects (the building blocks and the constructions obtained assembling them), to build conceptual structures (the meaning associated to each block, e.g. a math block, word block), and to compose actions (combination of output blocks like motors, LEDs, loudspeakers). Manipulating I-BLOCKS do not only mean constructing physical or conceptual structures but composing actions for building complex behaviours. During our work, we have succeeded in refining the studies in both schools and hospitals, and in diverse cultures such as in Denmark, Finland, Italy and Tanzania. We have also found, that some technological developments are necessary to provide the seamless interface and creative construction possibilities, e.g. the new connection mechanism and the possibility for authoring the contents of the individual I-BLOCKS as presented here.

\section{I-BLOCKS}

The I-BLOCKS are hardware building blocks, which are currently embedded in LEGO Duplo® bricks. The electronics and connectors have been designed so as to facilitate communication and power sharing between I-BLOCKS when these are physically connected. Each I-BLOCK contains a PIC 16F876 28-pin 8-bit flash microcontroller, and metallic connectors for power sharing and communication. A 2x4 Duplo I-BLOCK contains 2 male communication connectors on the top surface, and 2 female connectors integrated in the PCB on the bottom (See Figure 1). The I-BLOCKS all have a programming connector on the main PCB inside, and this allows for easy reprogramming and/or updating of the I-BLOCKS software.

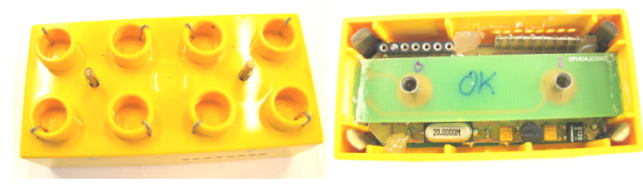

Figure 1 I-BLOCK top and bottom view

The communication protocol implemented is half-duplex, so that each $2 \times 4$ Duplo I-BLOCK can be connected to and communicate with up to 4 other I-BLOCKS. Power is supplied from special power IBLOCKS that are either power adapter supplied or 9V battery supplied and using the power sharing facilities of the I-BLOCKS, an entire structure of up to 20 I-BLOCKS can be supplied from a single 9V battery.

We developed a number of standard I-BLOCKS with the features mentioned above, and besides those we also developed some that have actuators built-in, such as DC-motors, servos, light, LCD-displays, sound, etc. and some that include sensors such as LDR resistors, microphones, flex sensors, touch and sonar (Ngo and Lund, 2004). Besides those, we have experimented with and build communication I-BLOCKS that use both IR and Radio communication to facilitate structure-structure communication (Ngo and Lund, 2004).

As each I-BLOCK includes its own microcontroller, the processing that goes on in a connected structure is truly distributed, and therefore the activity of the structure that is built is directly dependent on the physical properties of the structure.

\section{I-BLOCKS EXPERIMENTS}

We explored numerous strategies for structure dependent distributed processing on the I-BLOCKS platform. Different approaches have been taken to gain greater transparency for the user of the system, and to evaluate the areas of application for the I-BLOCKS. Some of the different experiments and their results are mentioned briefly below. 


\section{Arithmetic I-BLOCKS}

The arithmetic I-BLOCKS (Lund, 2003a) are meant as an educational tool, where the user physically builds binary expression trees (without having to know about those in order to build) using the standard operators addition, subtraction, multiplication and division. Here the standard I-BLOCKS without sensors or actuators (also called the CPU-blocks) are programmed to be either kind of arithmetic operator mentioned above. The user then decides the input to those arithmetic I-BLOCKS by using so-called user input I-BLOCKS, which can be set to any integer number between 0 and 99 .

\section{Neural I-BLOCKS}

The neural I-BLOCKS (Lund, 2003b, Nielsen and Lund, 2003) is a set of I-BLOCKS where the functionality of each I-BLOCK resembles that of a spiking neuron, so that the user here has to construct neural networks when designing a structure. In the Neural I-BLOCKS we have included the different sensor and actuator IBLOCKS, and it is hereby possible to build small robotic structures that will react on and possibly modify the surroundings. Besides, we also implemented Hebbian learning, which allowed for training of the built robot (Nielsen and Lund, 2003).

\section{Language I-BLOCKS}

In this experiment we implemented a grammatical checker to be used in language training in schools. Here each I-BLOCK is implemented as a word, and then the words have to be combined physically to form a sentence. The resulting sentence is then checked by a LCD I-BLOCK, and the sentence is displayed only if it is grammatically correct. Besides display output, we also implemented a sound I-BLOCK for playing a happy melody when the sentence is correct and a sad melody when wrong. The Language I-BLOCKS were tested in Danish and Italian schools and hospital, and proved to be very useful (Lund et al., 2003).

\section{The Living Tree}

The living tree (Marti et al., 2004) was developed together with master students from the University of Siena, and the platform implemented allows the users to express their state of mood through connecting I-BLOCKS of different characteristics and receiving different outputs (light, movement and sound) on that behalf. So in this experiment, it was up to the users to determine what combination of I-BLOCKS resulted in an output that was a reflection of their current mood state.

\section{The Toolset}

The new toolset borrows a lot of its functionalities from some of the other implementations mentioned above, and is a more general implementation, that allows the user to build and explore more freely. Basically, the toolset is an expansion of the arithmetic I-BLOCKS, where all sensor and actuator I-BLOCKS have been included to facilitate construction of different measuring tools and robotic structures. Generally, all of the sensor I-BLOCKS work as outputs only, meaning that they only receive input from their sensors, and they only transmit output on their communication ports. The actuators work in the exact opposite way, meaning that they only receive input on their communication ports and they only send input to their actuators. In this way it is up to the user to build paths between the right sensors and actuators using CPU I-BLOCKS with different functionalities. The different I-BLOCKS used in the toolset are presented in Table 1 below.

Table 1. The toolset of I-BLOCKS.

\begin{tabular}{|c|c|}
\hline & $\begin{array}{l}\text { CPU I-BLOCK } \\
\text { This I-BLOCK is the general processing I-BLOCK that is used for all kinds of } \\
\text { calculations - producing output from input received from one or more input ports. I- } \\
\text { BLOCKS of this type normally also contain } 8 \text { LEDs for producing light output, that can } \\
\text { either be used for run-time debugging or as a general actuator. }\end{array}$ \\
\hline & $\begin{array}{l}\text { LDR I-BLOCK } \\
\text { This I-BLOCK comes in two versions with either one or two LDR sensors. The LDR } \\
\text { sensor is a light sensor and senses the brightness of the light. }\end{array}$ \\
\hline & $\begin{array}{l}\text { Flex Sensor I-BLOCK } \\
\text { This sensor I-BLOCK is sensitive to touch, i.e. sensitive when the sensor is bent. }\end{array}$ \\
\hline
\end{tabular}




\begin{tabular}{|c|c|}
\hline & $\begin{array}{l}\text { Microphone I-BLOCK } \\
\text { This I-BLOCK comes in two versions with either one or two microphone sensors. The } \\
\text { microphone sensor is a sound sensor and senses the loudness of sounds. }\end{array}$ \\
\hline & $\begin{array}{l}\text { Sonar I-BLOCK } \\
\text { This I-BLOCK uses two sonar devices to determine distance to objects in front of them. } \\
\text { The one device is the sender and the other a receiver. It uses ultrasonic sounds to } \\
\text { calculate the distance. }\end{array}$ \\
\hline & $\begin{array}{l}\text { RFA I-BLOCK } \\
\text { This I-BLOCK can both be considered a sensor and an actuator I-BLOCK. It has a built- } \\
\text { in radio module which can be set up to either receive or transmit data. Besides, it also } \\
\text { has a built-in tilt sensor, that will output a value dependent on the I-BLOCKS angle } \\
\text { compared to water level. }\end{array}$ \\
\hline & $\begin{array}{l}\text { Button I-BLOCK } \\
\text { This I-BLOCK can be used to set values. When pushing the right button, the internal } \\
\text { number is increased } 1 \text {-by-1 until it reaches } 255 \text {, and the left button decreases the number. } \\
\text { It is easier to set the value, when this I-BLOCK is attached to the LCD I-BLOCK for } \\
\text { obtaining feedback. }\end{array}$ \\
\hline & $\begin{array}{l}\text { Switch I-BLOCK } \\
\text { This I-BLOCK can be used to set values. The value can be set between } 0 \text { and } 99 \text {. The } \\
\text { value can be read directly from the two turn-buttons. }\end{array}$ \\
\hline & $\begin{array}{l}\text { LCD I-BLOCK } \\
\text { This I-BLOCK is a general display I-BLOCK that can be used to display all sorts of } \\
\text { textual and numeric output. }\end{array}$ \\
\hline & $\begin{array}{l}\text { LEGO Motor I-BLOCK } \\
\text { This I-BLOCK takes input from any of the four communication connectors and converts } \\
\text { it into output for the standard LEGO Mindstorms motor. }\end{array}$ \\
\hline & $\begin{array}{l}\text { Sound I-BLOCK } \\
\text { This I-BLOCK has a built in beeper that can be used to produce sounds and melodies. }\end{array}$ \\
\hline & $\begin{array}{l}\text { Top Turned Servo I-BLOCK } \\
\text { This I-BLOCK can turn the top part of the I-BLOCK approximately } 90 \text { degrees in each } \\
\text { direction. }\end{array}$ \\
\hline
\end{tabular}

In April 2004 the toolset consisting of the I-BLOCKS mentioned above was tested at a workshop with students of Tumaini University, Iringa, Tanzania. Here we had pre-programmed all of the I-BLOCKS before taking them to Tanzania, and we wanted to test if they could be used as a tool for giving the students a handson introduction to IT and IT artefacts, and thereby making it easier for them to gain the concepts of e.g. inputs and outputs and sensors and actuators. The students were at first quite hesitant to play with the IBLOCKS, but after just 1-2 hours they started to find out what capabilities the different kind of I-BLOCKS had and how they could be combined to make small tools or robotic structures. Most of the first structures built by the students were direct connections of sensor and actuator I-BLOCKS, but after a presentation of our CPU I-BLOCKS, which we had pre-programmed with different functions, they started to realize that it was possible to process the input of one or more sensor I-BLOCKS before outputting it to an actuator IBLOCK. As a final project, the students brainstormed to designing and succeeding in building a wireless car burglar alarm that would tell the car-owner if somebody was touching his car while he was away from it.

The user experiments that have been carried out in this project and which are briefly explained above, have shown us that there are some limitations with the current version of the I-BLOCKS. First of all, the LEGO Duplo ${ }^{\circledR}$ housing does not allow for connections that are stable enough for e.g. robotic walking structures. In the current version, the I-BLOCKS structure simply disassembles when exposed to even light shakes. 
Another limitation of the current I-BLOCKS version is the lack of rotational information of the structure. It is e.g. impossible to record the precise 3D structure built, because the I-BLOCK-to-I-BLOCK connection does not carry any information about rotation. The gain of having this rotational information would be to have more possible states within each I-BLOCK so that each rotation angle could result in a different behaviour of the connected I-BLOCK and in the end the entire structure.

The I-BLOCKS are in the current version all hand-made, and therefore we have experienced some problems with especially the metallic connectors. Assembling and disassembling the I-BLOCKS several times results in lose connections and this problem should be solved in a new version.

Another issue is that the user until recently has been unable to alter the content of i.e. the CPU I-BLOCK. This limits the user to only be able to experiment with pre-programmed I-BLOCKS, and we might therefore be missing out on general functionalities that would enhance the entire system.

Finally, the current version of the I-BLOCKS is limited to the way one can build with the standard LEGO Duplo® I-BLOCK. The Duplo only has connectors on the bottom and the top, and therefore the direction of building almost always bottom-up. This problem will be investigated further in a section below.

In order to address the challenges, we engaged in the development of a simple graphical user interface for allowing users to author the contents of the individual I-BLOCKS, and we engaged in a design of 3D IBLOCKS with neighbourhood information. These two developments are described below.

\section{PROGRAMMING INTERFACE}

The I-BLOCK Application is a Java application that provides the user with a simple graphical interface to change the behaviour of an I-BLOCK. This application has been made to make it easy for the user to test her ideas, without having to ask a skilled programmer to make the programs. The user does not need to have any previous knowledge of traditional programming languages to use this interface and he needs only to have a concept of what inputs and outputs are, and what kinds of sensors and actuators the different I-BLOCKS contain (which a user normally obtains simply by playing with the I-BLOCKS, as exemplified with the Tanzanian project mentioned above). And finally the user needs to know how to connect the programming interface from a PC to an I-BLOCK.

\section{The Basics of the Application}

The I-BLOCK application integrates a database of I-BLOCKS, a database of functions and a database of programs with a graphic user interface. These databases are linked together, so that each I-BLOCK in the IBLOCKS database points to a set of functions in the functions database and a set of programs in the programs database. In this way, when a user selects a certain I-BLOCK from the selection list (as shown in Figure 2A) a specific number of programs are presented in the program list and a specific number of functions in the function list.

Now it is possible to select a program and upload this to the selected I-BLOCK. In order to upload a program, the PIC-programming device that we are currently using must be connected to a USB port and attached to the ISP port of the I-BLOCK in use.

Another option is to start building one's own program as shown in Figure 2B). Here functions can be inserted and connected to the inputs and outputs of the I-BLOCK, and hereby it is possible to build rather complex programs which can then be compiled and uploaded to the I-BLOCK. In the current experimental version we have only implemented the basic arithmetic operators and constants. Note also, that when an IBLOCK includes an actuator or a sensor, this is also represented as an output or input in the application.

The I-BLOCK application has been based on the functionalities of the I-BLOCKS toolset mentioned above, and the application has been tested October 2004 in an ICT course in Tumaini University, Iringa, Tanzania, where the students have successfully been using this interface together with the I-BLOCKS to gain the basic concepts of programming, before continuing on learning the Java programming language. The course started with an intensive two-days workshop playing and developing with I-BLOCKS, and then continued as a normal course with lectures and practical exercises each week, until a final project was performed by the university students, e.g. by teaching with I-BLOCKS at a secondary school in the rural area. Some of the outcomes of the first workshop were i.e. a tool that measures the height of people, an earthquake alarm, a burglar alarm and a vehicle that avoids walls. To verify the university students gain of 
knowledge about IT and technology, the students have been teaching secondary school children using IBLOCKS (Figure 3).

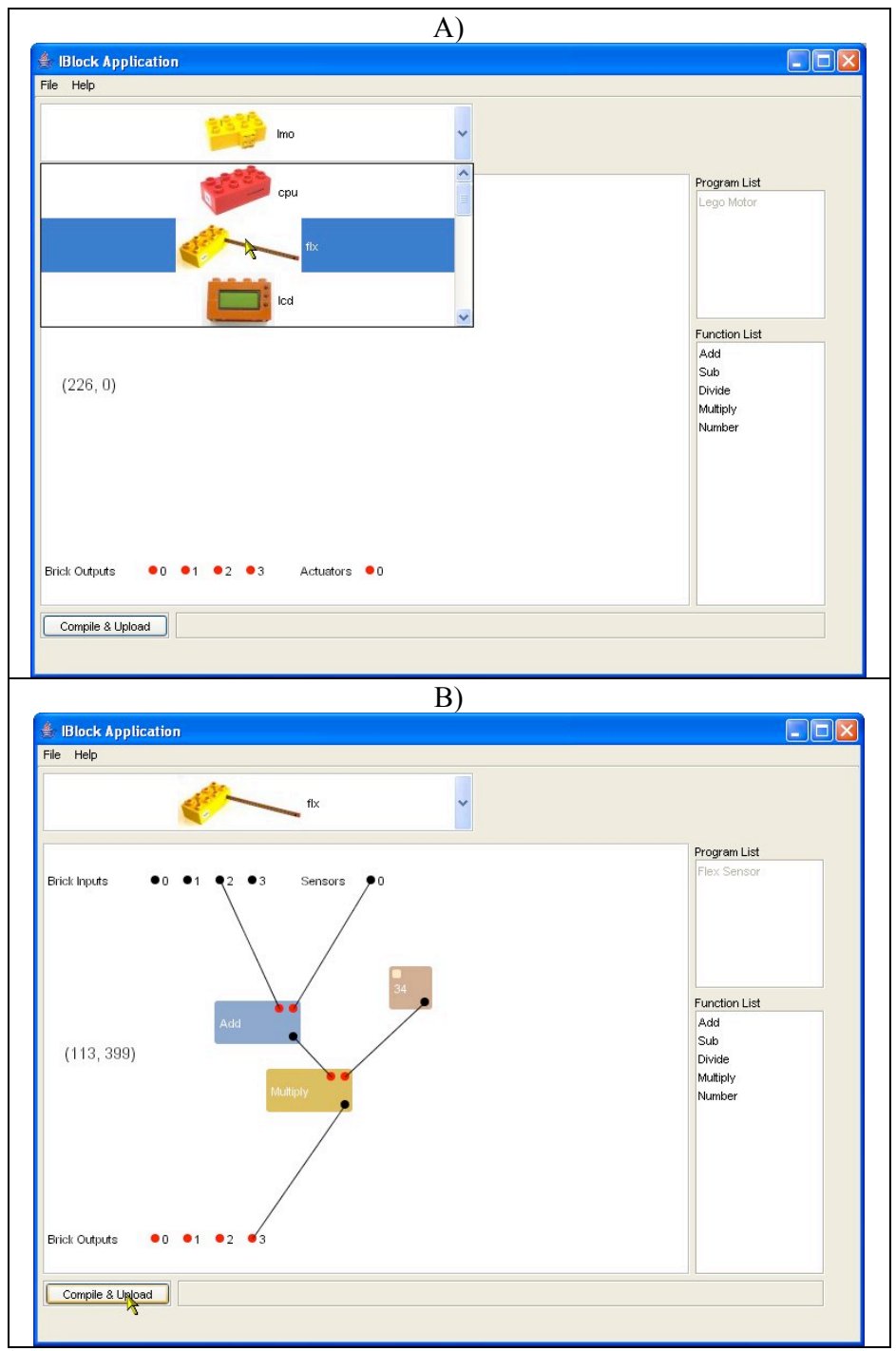

Figure 2. I-BLOCK Application features.

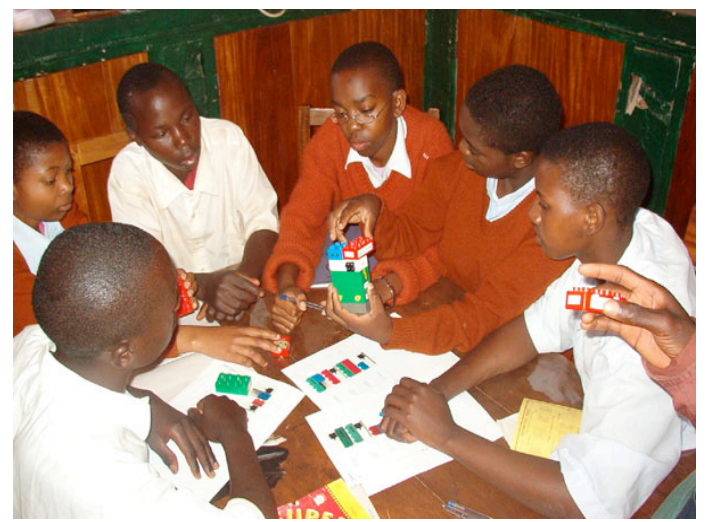

Figure 3. Some pupils from the Pommern Secondary School in Tanzania working with the I-BLOCKS 
During the Tanzanian workshop that initiated the full course, questionnaires were handed out, and two of the first questions give a good understanding of their gaining of the concept and what they have actually learnt. The answers were given as free text, but the two tables below describe the number of answers that fit within each mentioned category. The students were allowed to give several answers to each question.

What have you done for learning?

\begin{tabular}{|l|l|}
\hline General answers & $\#$ \\
\hline $\begin{array}{l}\text { I have been connecting I-BLOCKS to see and observe their individual and connected } \\
\text { functionalities and how/what they sense, actuate or process. }\end{array}$ & 19 \\
\hline $\begin{array}{l}\text { I have used I-BLOCKS and supplied software to download both pre-fabricated and self-created } \\
\text { programs. }\end{array}$ & 8 \\
\hline I have been taught about computer programming through the use of I-BLOCKS & 7 \\
\hline $\begin{array}{l}\text { I have tried to relate to what we have been doing in the workshop and how that can be used in our } \\
\text { daily life. }\end{array}$ & 3 \\
\hline I have built structures that allowed for arithmetic calculations. & 1 \\
\hline
\end{tabular}

What have you learnt?

\begin{tabular}{|l|l|}
\hline General answers & $\#$ \\
\hline $\begin{array}{l}\text { I have learnt how to use and program I-BLOCKS. (Both physical and computational } \\
\text { programming!) }\end{array}$ & 24 \\
\hline I have learnt how I-BLOCKS can be used many places in our daily life. & 9 \\
\hline I have learnt how the different I-BLOCKS can be used as teaching aids. & 6 \\
\hline I have practiced simple learning. & 1 \\
\hline I have learnt how to translate binary numbers into decimals through the I-BLOCKS & 1 \\
\hline $\begin{array}{l}\text { I have learnt, through I-BLOCKS that a computer program is an integration of small parts each } \\
\text { with different functions. And when combined you are able to perform more complex tasks. }\end{array}$ & 1 \\
\hline
\end{tabular}

\section{3D CHALLENGES}

In order to accommodate "real" 3D structures we have decided upon a cubic shape of the new I-BLOCKS. This shape is much more modular, and gives us the possibility of connecting up to 6 other I-BLOCKS to one I-BLOCK. However, when constructing this way, then one has to take gravity into account, (See Figure 4) and we will definitely experience problems such as bend-down and eventually self-disassembly when building perpendicular to the gravity vector without supporting the structure. This is, however, the case in all building kits on the market, and it just adds an extra challenge to the user of the system. When talking about self-disassembly we have to take into account that it will eventually occur and we need to design the connectors so that they will not break by such an incident.

Another challenge when building with cubes is that it might not be possible to connect cubes in e.g. corners (See Figure 4) if the connectors are outside the cube borders. So therefore we either have to design special integrated connectors, or the user will have to take this into account when building.
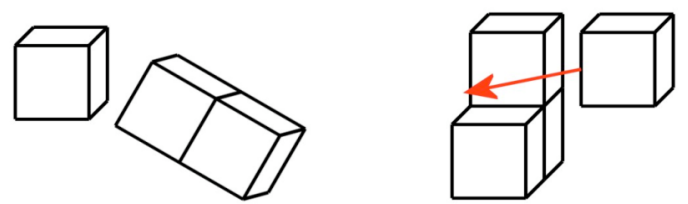

Figure 4. Structure self-disassembly and Corner connections

When building in 3D we would often like to know the precise 3D structure that has been built, because this information can directly be used to determine the functionality of the entire structure. The challenge here is to find ways to record this structural information, and likewise use this information in a clever way. New Connectors 
When designing connectors, there are a lot of things to take into account - both physical aspects and electrical aspects. In this section we will mention the different results we have reached during this process.

Starting with the electrical aspects of the connectors, we have to make sure that the following features are included in each connector side of the cube:

- Power transferral. Power should be able to be supplied from one I-BLOCK to another, so that it is possible to have only few special power bricks in the structure.

- Communication connectors. Each side should include one or two connectors for half or full duplex serial communication.

- Rotational information. In some way information must be present to determine all 4 possible connection angles when connecting the I-BLOCKS.

Also, when transferring power between the bricks, the electric connectors need to be able to transfer the sufficient amount of power, so that every I-BLOCK will work as intended.

Looking at the physical aspects of a connector, it is very important to look both at connectivity and productability. Also, there are such aspects as sex - do we want male-female connections or uni-sex. Different experiments have been carried out to find the optimal solution, and the results of some of those are shown below.

\section{Male-female system 1}

This connector uses an O-ring (not shown in Figure 5) as a connector between the male and female part. This type of connection is well tested in other applications, but in this case the O-ring is likely to fall of the male connector, when detaching it from the female. Because of our machining equipment it will also be difficult to make the connectors.

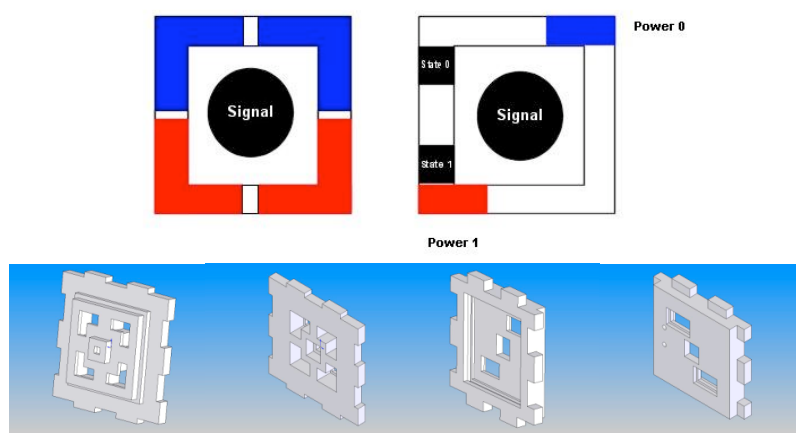

Figure 5. Electric and Physical Male-female connector with O-ring

Discovered advantages and disadvantages:

+ Easy to connect

+ Limitations with connecting at structure corners as explained previously.

- Not easy to produce

- There will be problems using O-rings.

Male-female system 2

This connector has a built-in "click" mechanism (Figure 6), which will lock the male connector to the female once connected. This type of connector is quite stable, but again we may have problems actually producing it, because of the limitations of our machining tool.

Discovered advantages and disadvantages:

+ Easy to connect

+ Simple

- Limitations with connecting at structure corners as explained previously.

- High demands to the material selected

- Complex to produce 

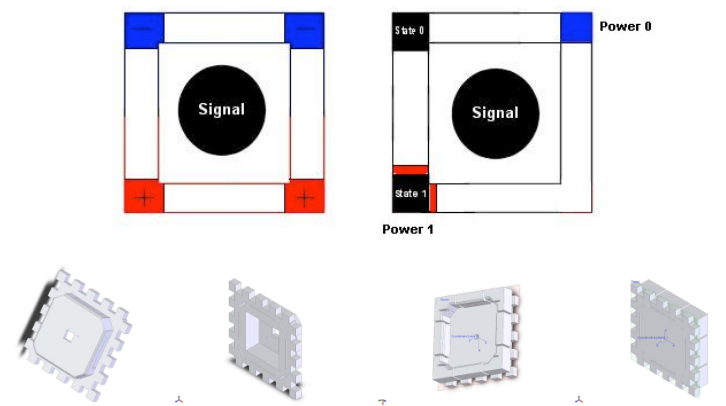

Figure 6. Electric and Physical Male-female "click" connector

Unisex system 1

This connector (Figure 7) is a unisex connector, and we were actually able to produce this connector with our machining equipment, but once produced, we discovered that the connectors were quite hard to join, and that there were slight misalignments once connected.

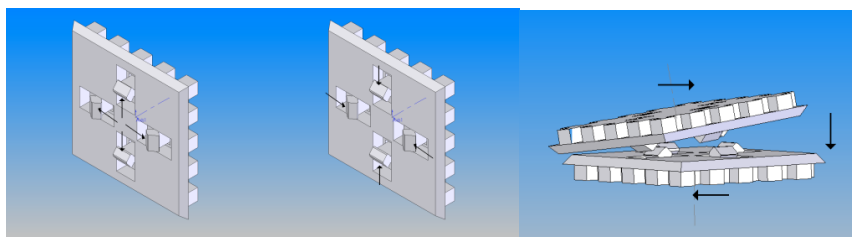

Figure 7. Unisex connector with flexible connectors

Discovered advantages and disadvantages:

+ More construction possibilities with unisex connectors

+ The connector is quite strong

- Physically connecting two connector surfaces is hard to do and takes a bit of practice

- Uncertain durability of the connectors

- Slight misalignment when connected

\section{Unisex system 2}

This system uses 8 connector pins on each connector side (Figure 8) and this ensures a very stable and strong connection. Likewise, the electrical connectors can be easily implemented in the connector.

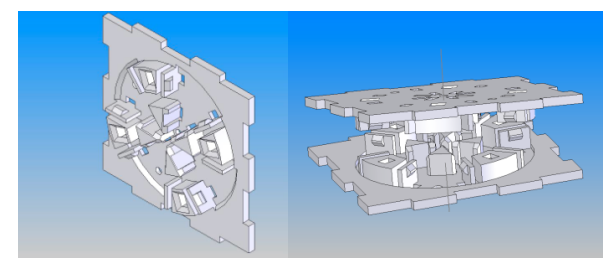

Figure 8. Unisex connector with circular connector

Discovered advantages and disadvantages:

+ More construction possibilities with unisex connectors

+ The connector is quite strong 
+ No manipulation of the connections

- Takes up a bit of space

The design that we decided upon using is the last one shown above. This design is quite feasible both for implementing the electrical connectors and also because of the great unisex features that really improve the construction possibilities. There are however still things that have to be tested in more prototypes, and we have to investigate what design is easier and cheaper to produce, while still providing the features that we need. We have currently produced 2 cubic new I-BLOCKS (Figure 9), and are as of this writing trying to find a stable way to join the individual sides of the cube and we are also in the phase of testing the electronics and trying to implement the metallic connectors as described in the next section.

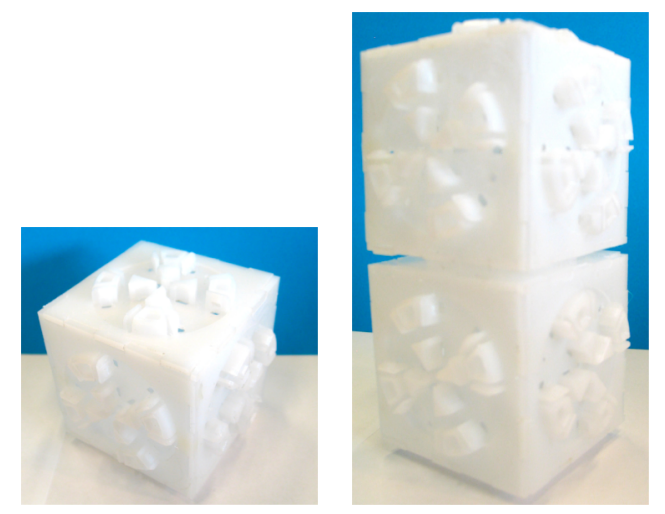

Figure 9. New I-BLOCKS - Single and Connected

\section{NOVEL HARDWARE}

The new hardware that we are testing currently to be used with the connectors mentioned above can be seen in Figure 10. The processor chosen is the Atmel AVR 8-bit Mega128 microcontroller which has 128kbytes of program flash and uses RISC architecture. This processor has been chosen to facilitate an increase in number of digital inputs and output pins necessary to support the new features of the I-BLOCKS and their connectors. The current side length of the new I-BLOCK is 5 centimetres. A new PCB has been made (Figure 10) which fits within the cube and allows for connection to all of the cube sides. Each cube side has one serial connector, power transferral capabilities and rotational information is gained from two spring connectors that connect to brass plates of high or low voltage on the adjoining connector as shown in Figure 10 .

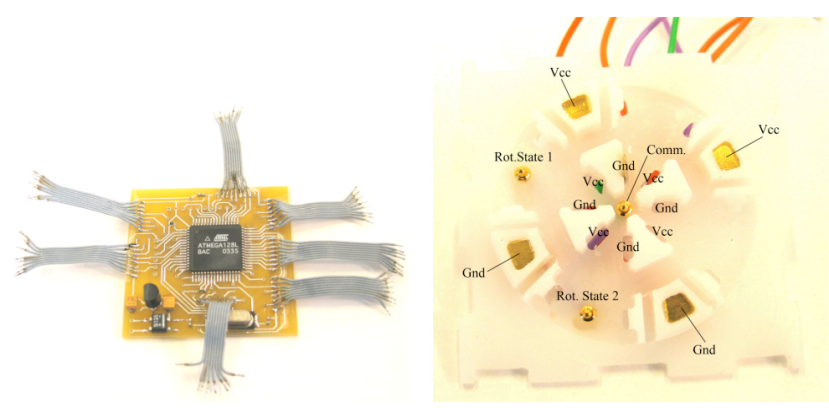

Figure 10. New PCB and new metallic connectors

The wire connections from one PCB to another was made, and tests have shown that communication, power transferral and rotation detection work as intended. 


\section{CONCLUSION}

Here, we developed a new graphic interface for authoring the contents of the individual I-BLOCK and we designed and developed a new cubic I-BLOCK with new connectors, as well as new hardware to support the new shape and capabilities of the I-BLOCK. During our work in both schools and hospitals, and in diverse cultures such as in Denmark, Finland, Italy and Tanzania we found that such technological developments are necessary to provide the seamless interface and creative construction possibilities. Therefore, this paper has focused on these necessary technological developments for improving the I-BLOCKS concept, and the development of novel I-BLOCKS based on this concept is ongoing.

In general, we find it important to develop such building devices able to simultaneously perform computations and to act as output devices of the intended functionality, since it allows us to advance constructionism by utilising the possibilities with the novel technology. We have throughout our work observed that the I-BLOCK is indeed a great tool for supporting different educational situations as well as children's play and general exploring curiosity. The development described in this paper will expand the possibilities within the concept even more and are likely to produce new uses of the I-BLOCKS, since it expands to 3D building, contents authoring, and easy manipulation.

\section{ACKNOWLEDGEMENT}

K. L. Jensen performed a lot of the developments for the 3D prototypes. C. Ryberg helped in technical development. M. Duveskog, M. Vesisenaho, and E. Sutinen, all University of Joensuu, collaborated on the IT training programme in Tanzania. The authors are grateful for the collaboration with Tumaini University and Pommern Secondary School, Tanzania.

\section{REFERENCES}

Camarata, K. Yi-Luen Do E., Gross M. D., and Johnson B.R., 2002. Navigational Blocks: navigating information space with tangible media, IUI 2002, 31-38.

Ishii, H., and Ullmer, B., 1997. Tangible Bits: towards seamless interfaces between people, bits and atoms. Proceedings of CHI '97, 234-241, ACM Press.

Lund, H. H., 2003a. Intelligent Artefacts. Sugisaka and Tanaka (eds.) Proceedings of 8th International Symposium on Artificial Life and Robotics. ISAROB, Oita.

Lund, H. H., 2003b. Neural Building Blocks. 1st International IEEE EMB Conference on Neural Engineering, IEEE Press, Italy.

Lund, H. H., Marti, P. and Palma, V., 2003. Educational Robotics: Manipulative Technologies for Cognitive Rehabilitation. Proceedings of 9th International Symposium on Artificial Life and Robotics. ISAROB, Oita.

Marti, P., Lund, H. H., Rullo, A., and Nielsen, J., 2004. Playing with Emotions. Proceedings of $12^{\text {th }}$ European Conference on Cognitive Ergonomics (ECCE'12), York.

McNerney, T., 2001. Tangible Computation Bricks: Building-blocks for Physical Microworlds. Proceedings of CHI 2001, ACM Press.

Nielsen, J. and Lund, H. H., 2003. Spiking Neural Building Block Robot with Hebbian Learning.Proceedings of IEEE International Conference on Intelligent Robots and Systems (IROS2003), IEEE Press.

Ngo, T. D., and Lund, H., 2004. Modular Artefacts. Position paper for ECOOP 2004 workshop: Components-oriented Approaches to Context-aware Computing, Oslo, Norway, June 14th, 2004. 